\title{
Spotify Tailoring for B2B Product Development
}

\author{
Abdallah Salameh \\ Computing, Science and Engineering \\ University of Salford \\ Salford, UK \\ a.salameh@salford.ac.uk
}

\author{
Julian M Bass \\ Computing, Science and Engineering \\ University of Salford \\ Salford, UK \\ j.bass@salford.ac.uk
}

\begin{abstract}
Agile software development has become increasingly common in the context of large-scale organisations. Typically, software organisations tailor agile methods to fit their needs and ultimately maximise success. The size of the organisation, business goals, and operative models are some examples of factors for which agile methods are tailored.

Spotify model is introduced to facilitate the development of a very large-scale project with a Business-to-Consumer (B2C) model, but mission-critical large-scale projects with Business-toBusiness (B2B) model are not addressed by the model. Hence, a question that imposes itself is: What are practitioner perceptions of agile tailoring when using the Spotify model?

In this paper, we conduct a longitudinal embedded case study to investigate practitioner perceptions of agile method tailoring on a large-scale mission-critical project in B2B environment. The case study lasted over 21 months during which 14 semi-structured interviews were conducted. To analyse the collected data, the Grounded Theory (GT) is adopted.

As a result, we identify 44 tailored practices and attributes for B2B product development. Based on this tailoring, 4 influential factors on "Spotify Tailoring" have been derived. These derived factors are worth considering for other organisations concerned with agile method tailoring for large-scale missioncritical projects in B2B context.
\end{abstract}

Keywords-Agile methods tailoring, Spotify tailoring, B2B, Large-scale, Mission-critical, Offshore, Outsource, Product Development, Longitudinal embedded case study

\section{INTRODUCTION}

Software development environments are complex and exhibit several challenges such as physical distances, time zones, culture differences, strategic issues, process differences, and knowledge management [1]. The introduction of agile methods shows a fundamental shift in how organisations try to cope with encountered complexity and volatility issues [2]. Agile methods are built around empowered and self-organising teams with a strong focus on communication and collaboration through various agile practices [3]. However, organisations tend to tailor agile methods to scale employed practices and to fit their needs best [3], [4].

Spotify model is originally introduced to facilitate the development of music streaming services in a very largescale $^{1}$ B2C project environment, which demands satisfying the needs of end-users [6]. The substantial notion of the Spotify model is to create autonomous yet collaborative squads [6]. Since Spotify values innovation, product development follows

\footnotetext{
1 "Very large-scale projects" have more than 10 teams [5]
}

Lean Startup methodology [6]. Spotify model does not provide guidelines for developing large-scale ${ }^{2}$ mission-critical financial projects in $\mathrm{B} 2 \mathrm{~B}$ environment [6]. B2B environment is deemed as complicated because of having conflicting agendas, politics and different priorities between two or more parties (organisations) [7]. Product development in B2B environment often requires the participation of many stakeholders from all businesses sides [7]. Those stakeholders at the supplier side should posses good industry knowledge to gain customers' trust. Thus, Product Owners (PO) and Key Account Managers (KAM) are expected to be the face of the product, lead innovation and help the customers (i.e., organisations that acquire a product or a software service from a supplier) to gain strong competitive advantage [7]. In this paper, we contribute to answer the research question: What are practitioner perceptions of agile tailoring when using the Spotify model in a large-scale mission-critical B2B project environment?

We carried out a longitudinal embedded case study in a very large-scale organisation, which has a large-scale missioncritical B2B project environment. We conduct direct observations over 21 months, during which 14 semi-structured interviews were conducted to find out how agile method tailoring is actually performed. Due to confidentiality agreement, a limited description of the explored project is provided. The Grounded Theory (GT) approach was adopted to analyse collected data. As a result, we identify 44 tailored practices and attributes, in terms of B2B product development, which are described by practitioners and identified during our observations. This "Spotify Tailoring" highlights 6 modified, 28 newly introduced, and 10 followed practices and attributes as originally introduced by Spotify model.

\section{SPOTIFY MODEL}

Spotify model is introduced to facilitate software development for hundreds of developers across 4 cities [6]. In Spotify, squads are autonomous but tightly aligned, and can use their own preferable agile methods [6]. The autonomy of the squads is manifested in their ability for bypassing layers of management. The alignment of squads refers to the extent to which the organisation strategy is proudly undertaken by having focused team interactions rather than tactics [6], [8].

\footnotetext{
2"Large-scale projects" have 2 to 10 teams [5]
} 
Product development in Spotify follows Lean Startup [6], [9]. When the organisation decides that it is worth moving forward, it builds a Minimum Viable Product (MVP), which is just enough to feel the narrative, but far enough for the feature to be complete. Afterward, the organisation releases the built MVP into production for few percent of the end users to start learning and analysing data. Thereby, it continues monitoring the data and tweaking the feature until it reaches the desired impact. As a result, the feature is gradually rolled out for all end users around the world. Spotify is not concerned with release planning and predictability but rather about innovation [6]. As long the organisation does not rollback a new feature and it reaches desired impact without considering specific velocity, then it is a success [6]. A $100 \%$ of predictability means $0 \%$ of innovation as Spotify focuses on business value delivery instead of plans fulfillment [6].

Spotify adopts fail-friendly environment by employing fast failure recovery strategy where some squads have a Fail Wall, which is shared with other squads to learn quickly and to eliminate such failures in future. To mitigate future risks, the organisation adopts a Postmortem Documentation process, which is performed at the end of projects, to determine what were successful or unsuccessful [6]. Thereby, the organisation tends to fix the process not just the product by capturing learning lessons to avoid the same problem in the future. To build experiment friendly culture and to minimise ripple effect of possible failure in releases, Spotify introduced a Limited Blast Radius technique [6]. It gives squads courage to do lots of small releases on a limited number of end-users to do experiments and learn quickly instead of wasting time predicting and controlling all risks in advance.

\section{Research Design And Methodology}

Our case study is carried out in a multinational very large organisation, which is a market leader in the financial industry. The company employs approximately 650 staff members in 60 markets and processes around 60 billion $€$ per year. This paper focuses only on one project, which manages missioncritical autonomous financial services. These autonomous systems are collection of connected sub-systems which operate under the control of one administrative software that presents a common defined management policy to the service. From the customers' point of view, this project is perceived as an offshore outsourced project based on Šmite's proposed terminology and taxonomy for GSD [10]. In this project, the developers are distributed over 6 co-located squads. Also, there are 3 KAMs, 5 POs ( 2 of them are empowered with KAM role), 1 architect, 2 agile coaches, and 1 test lead.

The research draws on direct observation of around 225 ceremonies over 21 months, and on conducting 14 semistructured interviews. The observed ceremonies include daily stand-ups, backlog grooming, planning sessions, and retrospectives. Direct observation provides deep understanding of the studied phenomenon and prevents any suspected deviation between "semi-structured interviews" view of matters and the "real" case [11]. To provide interviewees with the opportunity to raise any other issues, an open-ended guide was used. The questions were revised after the second interview. Each interview was recorded (around 50 minutes) and transcribed verbatim for detailed analysis in a continuous basis.

The GT (Glasserian approach) is employed to analyse collected data while having absence of up-front clear research problem [12]. This is achieved by harnessing a constant comparison of data at increasing levels of abstraction. In essence, this is a process of continuous undertaking of memoing, sorting, data collection, coding, constant comparison, and theoretical saturation. After each interview, the audio file and its written transcripts was carefully reviewed to ensure consistency. To facilitate the coding process a few questions suggested by Glaser were answered while conducting the open coding. Constant comparison was employed to refine the emerging categories from identified concepts. Moreover, the observations were analysed and compared to the concepts derived from the analysed interviews. We identified minor contradictions, which were explored and accommodated in the resulting grounded theory.

\section{FINDINGS}

A synergy has been discovered between the following categories (i.e., factors), which are depicted in Fig. 1, and influencing "Spotify Tailoring” in terms of product development for a large-scale mission-critical project in B2B environment.

\section{A. Strengthening project visibility for the customers}

1) Strengthening shared understanding of the business objectives: Establishing clear vision, by defining the scope and having a set of specifications, provides customers and squads with directions to avoid confusion during the development process. In fact, "project vision is communicated frequently, but it changes quite often. This is mainly due to the market demands since it is volatile."-P10, PO. Since the customers are scattered around the globe, they need to know what the target is. Therefore, the project vision, which provide long term direction is broken down by the POs into milestones, provides 1) the squads with short term motivation and guidance, and 2) meet the customers' needs. "Frequently communicating clear targets and establishing milestones based on the defined specifications is considered important for both of the squads and the customers"-P7, PO and KAM. To maintain shared project vision and specifications, the POs ensure in their regular meeting of 1) the ownership of the provided software service itself, and 2) the alignment of the product strategy and the overall roadmap of the organisation. "We have a regular weekly meetings between the POs to prevent the deviation from product's main purpose... the provided features should not only cover the needs of a specific customer but they should be also usable by all customers within a specific domain"-P12, PO.

2) Strengthening offshore B2B project management: Utilising globally accessible project management tools (e.g., Rally) and issue tracker tools (e.g., Jira) increase project visibility in B2B environment. Providing the customers with online Kanban boards results in effective management mechanism with 


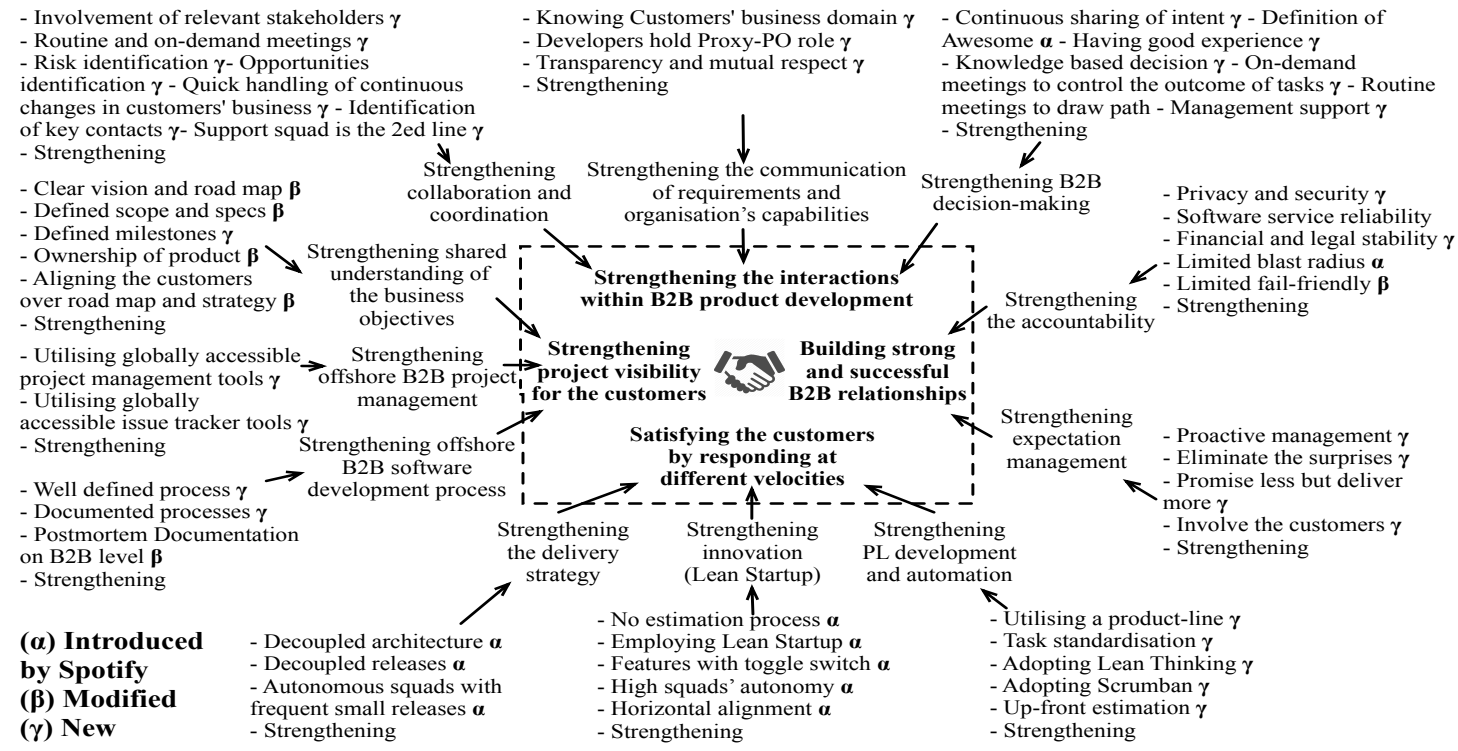

Fig. 1. Emergence of the influential factors on Spotify Tailoring (codes, concepts and categories - using the GT)

low coordination overhead. "To align B2B product development, we provide our customers with shared Kanban boards... This in turn facilitates the planning activities as customers can observe the progress and take decisions accordingly."-P11, KAM. Due to the strong culture of cross-pollination, all squads follow a standardised operational process that is controlled by Kanban and follows the employed definition of "DONE" "The definition of "DONE" ensures tasks' completeness, rules the overall process flow, and satisfies customers' needs"-P7, PO and KAM. Hence, the squads can respond to customers' needs in a highly transparent and disciplined manner.

3) Strengthening offshore B2B software development process: To improve project and process visibility in B2B environment, the organisation embraces well-defined and documented processes. "Continuously, we share with our customers feature instructions, release notes, and highlight on software development processes to streamline the business development"-P10, PO. Also, the organisation utilises Postmortem Documentation process, which is introduced by Spotify, to continuously document project related aspects, and to improve the development process. Therefore, "at the end of each small project, we get customer's feedback to improve the product and the process if needed"-P11, KAM.

\section{B. Strengthening the interactions}

1) Strengthening the collaboration and the coordination: Offshore outsourced software product development demands sufficient collaboration and coordination between the two sides of B2B. More complex issues in this mission-critical project require regular and on-demand involvement of multiple stakeholders from both sides. "Key players from both parties meet up on weekly basis to discuss the progress over an online Kanban board... we also meet up whenever is needed."P11, KAM. Being highly involved in the process keeps all sides informed, enables the identification of possible risks that both sides might overlook, and enables the identification of potential opportunities that might invest in. "The involvement of key stakeholders from both sides is considered crucial. Otherwise, we may realise that things should have been handled in different ways. Thus, our priorities are coming into focus or changing"-P7, $\mathrm{PO}$ and KAM.

The identification of key contact persons in the two sides strengthens the interaction in business and product development, and facilitates a continuous communication flow. POs and KAMs tend to "highlight on external communication hierarchy, internal structure, and squads' missions to make sure that all parties are on the same page"-P10, PO. Also, the support squad, which represents the second line of contact, is "continuously helping the customers, in a low Resolution Time, with service configuration management, issues investigation, requirements engineering, and continuously giving feedback to the POs and KAMs"-P14, Support Manager.

2) Strengthening the communication of requirements and the capabilities of the organisation: Product development is tightly linked with the continuous communication of requirements and the continuous communication of the organisation's (i.e., supplier) capabilities to the customers. Misunderstanding of the requirements or organisation's capabilities puts even simple implementations at serious risk.

Knowing customers' business domains facilitates precise interpretation of the requirements. When a customer requests a a new feature, "as much information as possible should be provided by the customer to speed up the support and the development processes"-P14, Support Manager. As new requested features are usually characterised with high uncertainty, developers take the role of Proxy-POs for the sake of requirement elicitation and to get the requested featured matured enough to start the development. "Usually, I get in touch with our customers to derive the requirements due to the 
vagueness of newly requested features"-P9, Senior Developer.

Both sides have different interests, but they overlap. The customers utilise the provided service to perform a business functionality that is outside their business domain. "A successful product development relies on fulfilling customers' needs and maintaining respect for what we need to accomplish"P10, PO. The organisation has developed a corporate culture based on transparency and mutual respect by "continuously communicating what capabilities (time and resources) we can provide within the upcoming period"-P11, KAM.

3) Strengthening B2B decision-making: The organisation emphasises on continuous sharing of intentions at different levels to facilitate decision-making. "As we are familiar with the intentions behind each component or feature, we are able to make the right decisions quickly"-P6, $\mathrm{PO}$ and KAM. Also, utilising the "Definition of Awesome", which is introduced by Spotify, in each area of the provided software service facilitates decision-making. "Possessing good knowledge about how the GOOD should looks like in all areas improves our product development"-P7, PO and KAM. In fact, decision-making is mostly shifted from a domain-based approach, which relies on routine decisions, to a knowledgebased approach, which relies on expertise. “Our POs' squad have long experience and knowledge in this industry, which in turn facilitates our work"-P12, PO.

\footnotetext{
"On-demand decision process is employed to control the outcome of tasks having high uncertainty "-P11, PO

"POs meet up regularly to ensure...and the ownership of our project as customers are enthusiastic about using our software service in their own way"-P11, PO
}

\section{Building successful B2B relationships}

1) Strengthening the accountability of the provided software service: The customers rely on this software service to offer their end-users wide range of payment solutions. Thus, the customers rely on the accountability of the organisation in question to provide stable, reliable, and secure financial software service, which impact the Return Of Investment (ROI). "If things go wrong with the provided software service, it will negatively affect customers' business, reputation, and revenues"-P11, KAM. Actually, "end-users assume that if a specific part of a customer's product is bad, then the whole product is bad too"-P13, KAM. Also, "the stability of the provided service is also about the financial and legal stability of the provided software"-P1, Agile Coach and Architect.

The organisation emphasises on continuously providing 24/7 stable software service. Instead of rolling out new implemented features to all customers at once, a "Limited Blast Radius technique is utilised to perform experiment on a limited number of end-users"-P8, Senior Developer. Consequently, the responsible squad decides whether to gradually rollout the new feature or roll it back based on the monitoring results.

The organisation employs a limited fail-friendly environment since it provides mission-critical software service. "As we provide financial software services, failure is not tolerated since it affects directly our reputation"-P1, Agile Coach and
Architect. However, failures can be encountered during the pilot launch of newly implemented features, which aim to improve and verify features' behaviours. These failures are utilised to learn and improve both of the process and the product. "In our squad-of-squads weekly meeting, we share the reasons behind encountered release issues to improve the product and the process if needed"-P12, PO.

2) Strengthening expectations management: Proactively managing the expectations builds strong B2B relationships. To achieve this, "a deeper understanding of the customers, at company and business domain levels, is considered important to provide customers with better solutions that would strengthen their competitive advantage"-P13, KAM. The organisation tends to eliminate possible surprises when working on tasks with high-level of uncertainty and complexity. "Announcing the initiation of implementation for a task brings customers' excitement and builds expectations. Not being able to deliver on time causes dissatisfaction and a loose of confidence in our ability to produce in the future."-P7, KAM. Hence, POs and KAMs tend to "always promise less but try to deliver even more-P5, PO. To mitigates possible dissatisfaction, POs and KAMs are continuously involving key stakeholders at the customer side in the process to eliminate possible misunderstandings. "When meeting customers, we try to avoid possible pitfalls by having a conversation in which both sides openly discuss what is expected from the other side to prevent possible misunderstandings"-P7, PO and KAM.

\section{Satisfying customers by responding at different velocities}

Since squads have different missions and adopt different tailored methods, they respond to customers' needs at different velocities. While some missions value innovation more than plan fulfilment, others value plan fulfilment more than innovation. We ignore strengthening the delivery strategy and strengthening innovation since they are covered by Spotify [6].

1) Strengthening product-line development and automation: The adoption of a product-line (PL) provokes task standardisation to streamline the development process. Since the project manages autonomous sub-systems, it "utilises a PL architecture, which facilitates the process of integrating the project into external sub-systems"-P9, Senior Developer. Eliminating waste, which is a key principle in Lean Thinking, is realised through the employment of predefined checklists. These "checklists are utilised to facilitate requirement extraction, code review, planning, estimation, documentation, knowledge sharing, etc"-P8, Senior Developer. This in turn, helps in automating and speeding up the development process.

Since such tasks are characterised with low degree of uncertainty, an up-front estimation process is considered beneficial. Those squads who work on the PL "employ some processes from Lean and Scrumban... we use bucket size, ondemand planning techniques, and average lead/cycle time"P5, PO. Hence, the POs are more confident to promise more predictable delivery deadlines. 
TABLE I

INFLUENTIAL FACTORS ON "SPOTIFY TAILORING"

\begin{tabular}{|l|l|c|c|}
\hline \multicolumn{1}{|c|}{ Factor } & \multicolumn{1}{|c|}{ Concepts / disciplines } & Spotify & Case Study \\
\hline \multirow{3}{*}{ Project visibility } & Shared understanding of the business objectives & $\approx$ Yes & Yes \\
\cline { 2 - 4 } & Globally accessible project management tools & No & Yes \\
\cline { 2 - 4 } & Employing offshore product development processes & No & Yes \\
\hline \multirow{4}{*}{ Employed interactions } & Collaboration and coordination & No & Yes \\
\cline { 2 - 4 } & Communication of requirements and the organisation's capabilities & No & Yes \\
\cline { 2 - 4 } & Facilitating decision-making & ₹es & Yes \\
\hline \multirow{3}{*}{ B2B relationships } & Accountability of the provided software service & Unknown & Yes \\
\cline { 2 - 4 } & Expectation management & No & Yes \\
\cline { 2 - 4 } The response time & Relying on the relationships among stakeholders & Yes & Yes \\
\cline { 2 - 4 } & Proper delivery strategy (based on the product-level and the mission) & Yes & Yes \\
\cline { 2 - 4 } & Uacilitating innovation by employing Lean Startup & No & Yes \\
\hline
\end{tabular}

Yes: covered, $\approx$ Yes: partially covered as depicted in Fig. 1, No: not covered, Unknown: no evidence

\section{Discussion AND CONCLUSION}

To maximise success in software development, organisations tend to tailor agile methods to fit their needs best. Spotify model is introduced to facilitate the development of a very large-scale project within B2C context [6], [8]. In fact, Spotify follows Lean Startup methodology for product development since it values innovation more than plan fulfilment [6]. The model as originally introduced cannot adequately serve largescale mission-critical projects in B2B environment without further adaptation and tailoring. Despite the fact that agile method tailoring has been a hot research topic in the agile community for some years now, limited scientific research has addressed the Spotify model [8].

Observing and collecting practitioner perceptions of how Spotify model is actually practiced in the industry can highlight important aspects that can be considered for agile tailoring. To this end, we conducted a longitudinal embedded case study in a very large-scale organisation, which has a mission-critical large-scale project with B2B environment. The study lasted over 21 months during which 14 semi-structured interviews were conducted to collect data. The collected data were analysed using the GT (Glasserian approach).

Our case study discovered 44 tailored practices and attributes by the organisation to fit for large-scale missioncritical $\mathrm{B} 2 \mathrm{~B}$ product development. These practices and attributes were classified into three main types, as follows:

1) Modified: 6 practices and attributes were modified to better fit the needs of the organisation under discussion.

2) New: 28 practices and attributes were newly introduced to cover some needs that are not considered by Spotify.

3) Already exist: 10 practices and attributes were followed as originally introduced by Spotify.

Based on our findings, we discovered a synergy between 1) the identified factors by our case study, and 2) influencing "Spotify Tailoring", in terms of product development. Table I presents 4 identified factors and their concepts in our product development case study. Each concept is supported by a set of practices and attributes as depicted in Fig. 1. The table also indicates the coverage of the adopted practices and covered attributes by the Spotify model and the organisation. Moreover, the table clarifies the extent of which Spotify model has been tailored in the organisation. These factors are worth considering by organisations having the same context and concerned with agile tailoring.

To gain more confidence in the results, we aim to conduct a comparative study. The intended study will investigate the influential factors on "Spotify Tailoring" by highlighting modified and newly introduced practices and attributes.

\section{REFERENCES}

[1] M. Niazi, S. Mahmood, M. Alshayeb, M. R. Riaz, K. Faisal, N. Cerpa, S. U. Khan, and I. Richardson, "Challenges of project management in global software development: A client-vendor analysis," Information and Software Technology, vol. 80, pp. 1 - 19, 2016.

[2] T. Dybå and T. Dingsøyr, "Empirical studies of agile software development: A systematic review," Information and Software Technology, vol. 50, no. 9, pp. $833-859,2008$.

[3] P. J. Ågerfalk, B. Fitzgerald, H. Holmström Olsson, and E. Ó Conchúir, "Benefits of global software development: The known and unknown," in Making Globally Distributed Software Development a Success Story, Q. Wang, D. Pfahl, and D. M. Raffo, Eds. Berlin, Heidelberg: Springer Berlin Heidelberg, 2008, pp. 1-9.

[4] A. S. Campanelli and F. S. Parreiras, "Agile methods tailoring a systematic literature review," Journal of Systems and Software, vol. 110, pp. $85-100,2015$.

[5] T. Dingsøyr, N. B. Moe, T. E. Fægri, and E. A. Seim, "Exploring software development at the very large-scale: A revelatory case study and research agenda for agile method adaptation," Empirical Softw. Engg., vol. 23, no. 1, pp. 490-520, Feb. 2018.

[6] B. Linders, "Don't copy the spotify model," October, 2016. [Online] Available: https://www.infoq.com/news/2016/10/no-spotify-model

[7] T. Sauvola, L. E. Lwakatare, T. Karvonen, P. Kuvaja, H. H. Olsson, J. Bosch, and M. Oivo, "Towards customer-centric software development: A multiple-case study," in 2015 41st Euromicro Conference on Software Engineering and Advanced Applications, Aug 2015, pp. 9-17.

[8] A. Salameh and J. Bass, "Influential factors of aligning spotify squads in mission-critical and offshore projects - a longitudinal embedded case study," in Product-Focused Software Process Improvement, M. Kuhrmann, K. Schneider, D. Pfahl, S. Amasaki, M. Ciolkowski, R. Hebig, P. Tell, J. Klünder, and S. Küpper, Eds. Cham: Springer International Publishing, 2018, pp. 199-215.

[9] R. Kuusela and M. Koivuluoma, "Lean transformation framework for software intensive companies: Responding to challenges created by the cloud," in Software Engineering and Advanced Applications (SEAA), 2011 37th EUROMICRO Conference on. IEEE, 2011, pp. 378-382.

[10] D. Šmite, C. Wohlin, Z. Galviņa, and R. Prikladnicki, "An empirically based terminology and taxonomy for global software engineering," Empirical Software Engineering, vol. 19, no. 1, pp. 105-153, Feb 2014.

[11] H. Robinson, J. Segal, and H. Sharp, "Ethnographically-informed empirical studies of software practice," Information and Software Technology, vol. 49, no. 6, pp. 540 - 551, 2007.

[12] J. Corbin, A. Strauss, and A. L. Strauss, Basics of qualitative research., 4th ed. Sage, 2014. 